bioRxiv preprint doi: https://doi.org/10.1101/2021.08.24.457368; this version posted August 26, 2021. The copyright holder for this preprint

(which was not certified by peer review) is the author/funder, who has granted bioRxiv a license to display the preprint in perpetuity. It is made available under aCC-BY-NC-ND 4.0 International license.

\title{
Integrated single-cell analysis of enteric glial cells reveals a molecular basis for postnatal neurogenesis and its therapeutic application
}

Richard A. Guyer ${ }^{l}$, Sukhada Bhave ${ }^{l}$, Rhian Stavely ${ }^{\text {, Ryo Hotta }}{ }^{1}$, Nicole Bousquet ${ }^{1}$, Meredith Omer ${ }^{\text {, }}$ Allan M. Goldstein ${ }^{1,2}$

Affiliations:

1 - Department of Pediatric Surgery, Massachusetts General Hospital, Boston MA

2 - Corresponding author:

Allan M. Goldstein, MD

Massachusetts General Hospital

55 Fruit Street, Warren 1151

Boston, MA 02114

Email: agoldstein@partners.org

Phone: 617-726-0270 


\section{Significance}

The enteric nervous system is comprised of neurons and glial cells derived from neural crest precursors that migrate along the gastrointestinal tract during embryonic development. Considerable efforts have been dedicated to exploring enteric neurons, revealing many subtypes based on structure, function, and molecular profile. However, enteric glial cells have yet to be fully characterized. We performed singlecell transcriptional and epigenetic profiling of thousands of enteric glial cells from adolescent mice, providing for the first time a multimodal atlas of these cells. We identify multiple subpopulations of enteric glia and propose a molecular basis for these cells' ability to generate neurons. Enteric glial cells closely match embryonic neuronal precursors and may be suitable for therapeutic neuronal regeneration.

\section{$\underline{\text { Abstract }}$}

Traditional models posit that enteric neurons and glial cells represent distinct terminal lineages derived from a common neural crest precursor. This model, however, does not explain the neurogenic capability of murine postnatal enteric glial cells. To characterize the full diversity of myenteric glial cells and identify a basis for the glial-to-neuronal transition, which we demonstrate using a two-marker system, we applied single-cell RNA sequencing and single-nucleus ATAC sequencing to generate a multiomic atlas of Plp1-expressing glial cells from the small intestine of adolescent mice. We identify nine transcriptionally distinct subpopulations of enteric glial cells, including cells expressing both canonical neural stem cell genes and enteric neuronal transcriptional factors. We refer to these Plp1positive cells with neural stem cell features as glial neuroblasts. Surprisingly, most glial cells maintain open chromatin at neuronal-associated loci, suggesting enteric glia are primed for neuronal transition. Comparison with the developing embryonic enteric nervous system shows postnatal glial cells maintain a transcriptional program closely matching embryonic neuronal progenitors. Transcription factor motif enrichment analysis and regulon analysis implicate AP-1 transcription factors in maintaining the glial neuroblast gene program. Three-dimensional cultures of postnatal enteric nervous system cells, which are enriched for glial neuroblasts and provide a niche for neuronal development, recapitulate the transcriptional changes seen during embryonic enteric neurogenesis. snATAC analysis shows chromatin closing consistent with terminal differentiation as glial cells become neurons in three-dimensional culture. In conclusion, postnatal myenteric glial cells include a neuroblast population and maintain a chromatin structure primed for neuronal fate acquisition.

\section{Introduction}

The enteric nervous system (ENS) consists of neurons and glial cells that are predominantly derived from migrating vagal neural crest progenitor cells during development of the gastrointestinal tract (1). Congenital or acquired loss of enteric neurons is a significant cause of disease $(2,3)$. Neurointestinal diseases may be amenable to cell-based therapies, but such an approach requires safe, large-scale delivery of enteric neurons or neuronal progenitors to the correct anatomic locations (4). The enteric glial cell (EGC) population has been shown to contain cells with neurogenic potential and is a potential source of autologous neuronal precursors for use in regenerative cell-based therapies (5-9). We undertook the present study to generate a transcriptional and epigenetic atlas of postnatal EGCs and evaluate their suitability as a therapeutic product for neurointestinal disorders.

Postnatal enteric glial cells in the myenteric ganglia have not been characterized jointly at the transcriptional and epigenetic levels, leaving the broad term EGC to be used to refer to a diverse population of cells. The fact that the expression of postnatal glial markers, including Sox10, Proteolipid 
protein 1 (Plp1), Glial fibrillary acidic protein (Gfap), and S100b, do not perfectly overlap in the adult mouse ENS indicates heterogeneity and suggests distinct functional states $(10,11)$. While differences in marker detection could arise from inefficient staining or from transient variations in protein expression, other signs of glial diversity include heterogeneous responses to purinergic stimulation (11), dye coupling of only a subset of glial cells within ganglia (12), and preferential stimulation of Gfap transcription in response to lipopolysaccharide exposure (13). Recent scRNA seq studies have reported multiple clusters of glial cells based on differential expression of highly variable genes in both humans (14) and mice (15), but in-depth analysis of these cells has not been reported. As a result, the number of glial cell states in the myenteric plexus remains unknown, as do the transcriptional circuitries underlying different glial programs.

According to the commonly-held model of ENS development, a common neural crest-derived progenitor population invades the developing intestine, proliferates, migrates through the gut, and ultimately differentiates along distinct glial and neuronal lineages $(1,16-18)$. This model is supported by experiments showing neural crest-derived cells within the embryonic gut that express both glial and neuronal transcription factor genes - such as Sox 10 and Phox $2 b$, respectively - in developmental model systems $(18,19)$. Because Sox 10 expression precedes and is necessary for expression of neuronal fatedetermining genes such as Phox $2 b$ and Ret (20,21), Sox10-expressing cells must include neuronal precursors. It is not clear, however, that postnatal Sox 10-positive glial cells represent a distinct cell fate decision. Arguing against a distinct, terminal glial fate is accumulating evidence that postnatal glial cells generate neurons and maintain proliferative capacity $(5,6,8,9,22)$. There is considerable overlap between genes marking ENS progenitors during prenatal development and those denoting postnatal glial cells, such as Sox10, Sox2, Ngfr, and Ednrb. This raises the possibility that postnatal glial cells are phenotypically indistinguishable from the embryonic precursors of enteric neurons, and they may represent the same cell population.

In the present study, we apply single-cell RNA sequencing (scRNAseq) and single-nucleus assay for transposase-accessible chromatin sequencing (snATACseq) to build a transcriptional and epigenetic atlas of myenteric glial cells in the adolescent mouse intestine. We hypothesize that postnatal myenteric glial cells maintain a transcriptional program similar to embryonic ENS progenitor cells, and that their chromatin structure permits rapid neuronal differentiation. We compare our data with published scRNA seq data derived from the prenatal enteric nervous system of mice and humans. We show that postnatal enteric glial cells include persistent, incompletely differentiated neuronal progenitor cells we call glial neuroblasts, as well as populations expressing genes that suggest functions related to extracellular matrix remodeling, extracellular fluid ion balance, and environmental sensing. Moreover, we demonstrate that glial neuroblasts can be massively expanded in suspension culture and differentiate into neurons along a pathway that recapitulates embryonic enteric neurogenesis. Our glial cell atlas reveals the diversity of postnatal glial cells and suggest autologous glial cells have great potential as a therapeutic product for neurointestinal diseases.

\section{Materials and Methods}

\section{Animals}

This study was performed according to experimental protocol 2009 N000239 approved by the Massachusetts General Hospital Institutional Animal Care and Use Committee. Plp $1:$ GFP mice were kindly gifted to our laboratory by Wendy Macklin (23). Actl6b::Cre (stock number 027826) and (R)26tdTomato (stock number 007914) mice were purchased from Jackson Laboratories (Bar Harbor, ME) $(24,25)$. Dual reporter mice were generated by first crossing Actl6b::Cre animals with (R)26-tdTomato 
animals, and subsequently crossing offspring with $P l p 1:: G F P$ animals to yield Plp $1:$ GFPI/ Actl6b::Cre;(R)26-tdTomato offspring.

\section{Isolation of ENS Cells}

Mice of the indicated ages and genotypes were euthanized and their small intestine was removed from duodenum to terminal ileum. The longitudinal muscle-myenteric plexus (LMMP) layer, which contains myenteric ganglia, was carefully dissected from underlying tissue under a dissecting microscope in icecold PBS supplemented with 10\% bovine serum albumin. After dissection LMMP tissue was digested for 60 minutes at $37^{\circ} \mathrm{C}$ in dispase $(250 \mu \mathrm{g} / \mathrm{ml}$; STEMCELL Technologies, Vancouver, BC) and collagenase XI (1mg/ml; Sigma-Aldrich, St. Louis, MO). Following digestion, the cells were filtered via a 40 micron filter to ensure a single-cell suspension and either taken immediately for sorting or placed in suspension culture.

\section{Cell Sorting}

Cell sorting was performed by staff at the Harvard Stem Cell Institute's Center for Regenerative Medicine Flow Cytometry Core facility located on the Massachusetts General Hospital campus. Sorting was performed on BD Bioscences (Franklin Lakes, NJ) FACSAria sorting instruments.

\section{Suspension Cultures}

Immediately after digestion and filtering, cells were counted and resuspended at a density of $10^{5}$ cells/ $\mathrm{mL}$ in a 1:1 mixture of DMEM (Thermo Fisher, Waltham, MA) and NeuroCult Basal Media (STEMCELL Technologies, Vancouver, BC) supplemented with $20 \mathrm{ng} / \mathrm{mL}$ FGF, $20 \mathrm{ng} / \mathrm{mL}$ IGF1, 2\% B27 supplement, $1 \% \mathrm{~N} 2$ supplement, $50 \mu \mathrm{M} \beta$-mercaptoethanol, and $75 \mathrm{ng} / \mathrm{mL}$ retinoic acid. $10^{5}$ cells/ $\mathrm{mL}$ in a total volume of $10 \mathrm{~mL}$ were placed $10 \mathrm{~cm}$ flasks (Corning Inc, Corning, NY) at $37^{\circ} \mathrm{C}$ and $5 \%$ $\mathrm{CO}_{2}$ for 10 days. Media was replaced on day 5 by centrifuging cells at $250 \mathrm{~g}$ for 3 minutes followed by resuspension in the same media and return to the same flasks and incubator conditions for 5 more days. Mice age 12-16 weeks were used to obtain cells for suspension cultures.

\section{Preparation of scRNAseq Libraries}

For P14 mice, LMMP cells were isolated from Plp $1::$ GFP mice and sorted for GFP+ cells as described above. Immediately after sorting, cells were manually counted with Trypan blue to assess viability. Cells were then delivered immediately to the MGH NextGen Sequencing Core Facility and core staff prepared cDNA libraries using the 10X Genetics v3.0 kits. The standard 10X Genetics workflow was used. Cells from 4 mice were used in three batches, with the first batch containing pooled cells from 1 male and 1 female mouse and the remaining two batches each containing cells from a single mouse (1 male, 1 female). For scRNA seq of three-dimensional (3D) cultures, single cells were obtained by digesting cultures for 45 minutes with Accutase (STEMCELL Technologies, Vancouver, BC). Cells were then sorted as described above into GFP+ and GFP- populations and immediately returned to the laboratory where they were manually counted with Trypan blue to assess viability. After determining viable cell counts, a 10X Chromium Controller located in our facility was used along with 10X Genetics (Pleasanton, CA) v3.1 kits to generate gel bead emulsions (GEMs), followed by library preparation according to the manufacturer's protocol. Cells from 4 mice were used to generate 3D cultures for scRNAseq, with cells from 2 male mice and 2 female mice each pooled together and cultured separately.

\section{Preparation of SnATAC and Multiomic Libraries}

For adolescent mice, LMMP was isolated from P16-18 animals as described above and GFP+ cells were sorted. Cells from three male mice were pooled and then split into three samples, each of which was run on one lane of a GEM chip. 10X Genetics Multiomic kits were used, and GEMs were 
generated using the 10X Chromium Controller located in our facility. snATAC libraries were generated using the standard 10X Genetics workflow. For two of the samples, gene expression library generation failed. ATAC library generation was successful for all three samples. Two samples were thus limited to snATAC data, while the third was a multiomic library with gene expression and ATAC data from the same cells. For multiomic analysis of suspension cultures, LMMP cells from three 12-week-old Plp1::GFP mice were pool and grown in suspension culture conditions for 10 days. Cells were then dissociated as described above and sorted to isolate the GFP+ population. A single lane on a GEM chip was then used to generate GEMs. The 10X Genetics multiomic library preparation workflow was then undertaken in accordance with the manufacturer's protocol.

\section{Sequencing and Genome Alignment}

All sequencing was performed at the Harvard University Bauer Core Facility, where libraries were sequenced on either Illumina NextSeq or Illumina NovaSeq instruments. Demultiplexing, genome alignment, and feature-barcode matrix generation was performed with the 10X Genetics Cell Ranger software pipeline.

\section{Single Cell Data Analysis}

snRNA seq and snATAC seq data was analyzed with the open-source Seurat and Signac packages implemented in the R computing environment. For the adolescent glial scRNA seq dataset, cells more than one standard deviation away from the mean number of genes detected were filtered, as were cells with greater than 10\% mitochondrial genes. Datasets were integrated using the SCTransform workflow in Seurat (26). After integration, principle component analysis (PCA) was performed. Neighbors were identified and UMAP projection was performed using the first 30 principal components. Clusters were identified using the "FindClusters" command with resolution $=0.25$ using the Louvain algorithm (27).

snATAC data was processed using the standard Signac workflow. Briefly, cells with fewer than 1000 or more than 100,000 ATAC fragments were filtered, as were cells with nucleosomal enrichment $>2$ or transcriptional start site enrichment $<1$. Peaks within each replicate were identified using MACS2, and peaks were merged across datasets with the "reduce" function of the IRanges R package. Datasets were then merged. Dimensional reduction was performed with latent semantic indexing (LSI) via the "RunTFIDF" command, "FindTopFeatures" function with min.cutoff set to 5, and "RunSVD" function. UMAP projection was performed utilizing LSI components 2-50.

Label transfer between datasets by canonical correlation analysis was performed using the "TransferData" function in Seurat. Anchor cells for data transfer were identified using the "FindTransferAnchors" function with the "pcaproject" reduction option and log normalization. The top 30 principal components were used for the "TransferData" function.

Regulon analysis was undertaken using pySCENIC software (28). For each dataset analyzed, the 5000 most variable genes within the full dataset were identified by Seurat's "FindVariableFeatures" command. A gene expression matrix containing only these 5000 variable genes was then exported in CSV format and used as input for the pySCENIC pipeline. pySCENIC results were imported to Seurat as an assay object via the "CreateAssayObject" command.

Transcription factor motif enrichement was implemented with the ChromVAR software package implemented through Signac (29). JASPAR2020 Mus musculus core transcription factor motifs were utilized (30). ChromVAR results were imported to Signac as an assay object. 
RNA velocity analysis was performed using Velocyto (31) on the Mass General Brigham ERISOne Research Computing Cluster, with subsequent analysis performed using scVelo (32) in the Python environment. Datasets were filtered to include only cells use for prior analyses in the Seurat. Datasets were then normalized and scaled, and 5000 variable genes per dataset were retained for analysis. After recovering RNA dynamics, RNA velocities were calculated using the "dynamical” mode.

\section{Live Cell Imaging}

GFP+/tdTomato- cells were sorted from LMMP suspension cultures derived from Plp1::GFPI/ Actl6b::Cre;(R)26-tdTomato dual reporter mice. Cells were placed in adherent culture conditions in neuronal differentiation media, which consists of BrainPhys Neuronal Media ( STEMCELL Technologies, Vancouver, BC) supplemented with 1\% N2 supplement and 2\% NeuroCult S1 supplement (STEMCELL Technologies, Vancouver, BC). After one day media was removed and replaced with a thin layer of phosphate buffered saline. Cells were then imaged at 10x magnification using a Keyence microscope. After imaging, differentiation media was replaced and cells were returned to culture for 4 more days. Repeat imaging was performed after 5 days in culture.

\section{Published Data}

Data of the mouse embryonic ENS at P15.5 and P185 was published by Morarach et al (33). This data was obtained from the NCBI Sequence Read Archive. Runs SRR11635571, SRR11635572, and SRR11635573 were downloaded using the SRA Toolkit "fastq-dump" command. Genome alignment and feature-barcode matrix generation was performed with the Cell Ranger "cellranger count" command on the Mass General Brigham ERISOne Research Computing Cluster. Further analysis was performed with Seurat in the R environment. Briefly, the datasets were filtered as the authors describe in their paper, with removal of cells with $<1000$ genes detected, $>6000$ genes detected, $>40,000$ total RNA counts, and $>5 \%$ mitochondrial genes detected. Datasets were then integrated using the standard Seurat workflow. Following integration, PCA was performed and UMAP projection was undertaken with the top 30 principle components. Labels were transferred as described above. The human embryonic ENS data was published by Fawkner-Corbett et al (34). The gene expression matrix for ENS cells was downloaded from https://simmonslab.shinyapps.io/FetalAtlasDataPortal. We used the dimensional reduction and cell clustering performed by the authors.

\section{$\underline{\text { Results }}$}

scRNAseq and snATACseq reveal diversity among postnatal enteric glial cells.

We first sought to characterize glial cell diversity in the myenteric plexus of adolescent mice. After filtering low quality cells from our scRNAseq dataset, we identified 11 transcriptionally distinct clusters of Plp1-expressing enteric glial cells. Two clusters, each with $<100$ cells, expressed markers both of glial cells (Plp1, Sox 10) and of either neurons (Tubb3, Uchl1) or of smooth muscle cells (Acta2) (data not shown). Although prior work suggests glial cells can generate both neurons and myofibroblasts (5), we could not rule out the possibility of doublet contamination so these cells were excluded from further analysis, leaving 13,936 cells. The remaining cells included 9 clusters based on differential expression of highly variable genes (Fig 1A, 1B). Hierarchical clustering of pseudobulk replicates identified clusters 8 and 5 as being highly similar to one another and relatively dissimilar to other clusters (Fig 1B); these clusters showed high expression of S phase- and G2/M phase-associated genes and thus represent replicating cells (SI Appendix, Fig S1A, S1B). Clusters 0 and 6 had very similar transcription to one another. Cluster 2 also has a similar transcriptional program as clusters 0 and 6, with the addition of genes associated with response to environmental pathogens (Fig 1B and SI Appendix, Fig S1C), suggesting a role in pathogen sensing and host defense. Consistent with prior bulk RNA sequencing studies (10), we found that extracellular matrix proteins, including collagens and 
proteoglycans, are expressed in most glial cells, as are voltage-gated potassium channels (SI Appendix, Fig S1D).

Cluster 4 has a very distinct gene expression pattern, and clusters 3 and 1 also expressed cluster 4 marker genes, albeit at lower levels than cluster 4 (Fig 1B). To test whether cluster 4 cells represent a distinct class of glial cell, we examined transcript levels of known glial markers (Plp1, Sox10, Sox6, Fbxo7, S100b, Gfap), neural stem cell-associated genes (Foxo1, Sox2, Tgfb2, Cdh2), and transcription factors with known roles in enteric neurogenesis (Hand2, Tbx3, Phox 2b). Clusters 3 and 4 displayed a distinct pattern, with relatively low expression of Plp1, Sox 10, and Sox6, as well as being the only glial clusters with detectable Gfap transcripts. Clusters 3 and 4 were also notable for high expression of neural stem cell genes, such as Sox 2 and Cdh2, and the enteric neuron-associated factors Hand2, Tbx3, and Phox $2 b$ (Fig 1C). Cluster 1 also displayed higher expression of several of these genes relative to other glial groups (Fig 1C), although not to the same degree as cluster 3 and 4. We thus speculated Cluster 4 represents cells primed for neuronal differentiation and we henceforth refer to them as "glial neuroblasts." Based on their transcriptional profile, we refer to clusters 1 and 3 as "transitional glia," since they appear to be at an intermediate state between glial neuroblasts and other glial cells.

RNA transcription can change rapidly and unpredictably within cells, and scRNAseq is susceptible to drop-out effects and other measurement errors. To confirm the diversity we observed was not due to transient variations or to measurement artifacts, we undertook snATACseq of glial cells from P16-18 mice. Cells from three mice were pooled, and three batches of these pooled cells were used for library generation and sequencing. After filtering low-quality cells and removing small clusters of presumed contaminant cells, a dataset of 10,748 single nucleus ATAC measurements remained. The results suggested a similar level of postnatal glial diversity as scRNAseq, with eight clusters identified based on chromatin accessibility patterns (Fig 1D). snATACseq cluster 3 showed a nearly inverse chromatin pattern compared to clusters 4 and 5, suggesting distinct biological functions for these groups of cells (Fig $1 \mathrm{E}$ and $1 \mathrm{~F})$. We again noted several clusters $(0,1,2$, and 6$)$ that are very similar to one another with respect to ATAC signal (Fig 1E). These highly similar clusters appeared to exist at an intermediate state between cluster 3 on the one hand and clusters 4 and 5 on the other, with open chromatin at ATAC peaks associated with both of these distinct groups, albeit with lower signal intensity (Fig 1E). This was illustrated by chromatin accessibility at loci on chromosomes 3 and 7 that are markers of cluster 3 and cluster 4, respectively (Fig 1F). Cluster 9 was a small cluster with a unique chromatin signature and exhibited more variation between replicates (Fig 1E).

\section{Multiomic sequencing of enteric glial cells uncovers the relationship between chromatin structure and gene expression}

We used a multiomic approach to obtain both gene expression and chromatin accessibility data from the same cells. We examined the expression of glial markers, neural stem cell-associated genes, and enteric neuronal transcription factors in these cells and found that snATACseq clusters 4 and 5 have a similar gene expression pattern as scRNAseq clusters 3 and 4, with high expression of neural stem cell genes and neuronal transcription factor genes and lower expression of Plp1 and Sox 10 than other glial cells (Fig 1G). To globally assess similarity of gene expression programs, we used canonical correlation analysis to transfer cluster identities from our scRNAseq dataset to the 3531 cells for which we had multiomic data. Label transfer from scRNAseq data to multiomic cells assigned great majority, including nearly all cells in snATACseq clusters $0,1,2,3$, and 6 , to scRNAseq cluster 0 (Fig $1 \mathrm{H}$ and 1I), thus identifying an epigenetic signature and gene expression program that predominates among the majority of postnatal glial cells. Because the epigenetic pattern in these cells is intermediate between the more distinctive cluster 3 and clusters 4 and 5, we speculate they have the capability of adopting either fate and refer to them collectively as "plastic glia." 
We observed that snATACseq clusters 4 and 5, which have a distinct chromatin profile relative to other glial cells (Fig 1E anf 1F), scored very highly for the gene expression program of scRNAseq clusters 4 and 1, respectively (Fig 1H and 1I). snATACseq cluster 5 also scores very highly for scRNAseq cluster 3 (Fig 1H and 1I). We thus referred to snATACseq cluster 4 cells as "glial neuroblasts" and to snATACseq cluster 5 cells as "transitional glia." Consistent with the gene expression data, signals at ATAC peaks in the transitional glial cluster are intermediate between those of glial neuroblasts and the plastic glial cells (Fig 1E).

Interestingly, snATACseq cluster 3 was the only ATAC cluster to score highly for the scRNAseq cluster 6 transcriptional program (SI Appendix, Fig S2). We also noted that many ATAC peaks with reduced signal in snATACseq cluster 3 are at loci displaying increased signal in scATACseq clusters 4 and 5 (Fig 1E), suggesting that snATACseq cluster 3 cells are restricted from adopting the glial neuroblast fate. As a consequence, we referred to these cells as "restricted glia." While we could not be certain of the epigenetic state of cells in our scRNAseq dataset, we noted that snRNAseq cluster 6 shares marker genes with these restricted glial cells (SI Appendix, Fig S3), so we denoted scRNAseq cluster 6 cells as "restricted glia" as well. Finally, a small number of cells in snATACseq cluster 9 scored highly for a genetic program associated with DNA replication in our scRNAseq data (SI Appendix, Fig S2). We speculate the epigenetic changes in cluster 9 reflect chromatin accessibility mediated by DNA replication machinery.

Glial cells maintain accessible chromatin at loci of neuronal fate-determining transcription factors, but factor expression correlates with distal elements

We assessed the mean ATAC signal at the transcriptional start site (TSS) for 274 glial marker genes and 317 neuronal marker genes culled from a published dataset (15). As expected, glial cells enrich for ATAC signal at the TSS of most glial marker genes (Fig 2A). However, we also found accessible chromatin at a surprising number of neuronal marker gene TSSs (Fig 2A). To explore this further, we focused on transcription factors known to determine neuronal fate that are highly transcribed in neuroblasts and preneuroblasts as compared to other glial cells: Tbx3, Hoxa4, and Phox2b (Fig 1C, Fig 2B). Interestingly, all three of these factors have similarly-accessible TSS and promoter regions across all snATAC clusters (Fig 2C, 2D). To confirm accessible chromatin is not a general feature of TSS loci in glial cells, we examined the Alb and $H b a-a l$ loci, which encode genes without known neuronal or glial functions, and saw no pattern of open chromatin at the TSS, within the promoter region, or within the gene body (SI Appendix, Fig S4). We hypothesized that glial cells are epigenetically poised to transcribe neuronal fate-determining transcription factors. Additionally, we suspected expression of these neuronal genes is controlled by enhancer selection. We then examined TSS accessibility at neuroblast marker gene loci, as well as snATAC signal at peaks linked with expression of neuroblast marker genes. Consistent with our hypothesis, most neuroblast gene loci have accessible TSS regions in all glial clusters, although the signal is strongest in glial neuroblasts (cluster 4) and transitional glial cells (cluster 5). In contrast, putative enhancers linked with gene expression display markedly heightened signal in glial neuroblasts and transitional glia relative to other cell clusters (Fig 2E). This is illustrated by the Tbx3 locus, where transcriptional activity corresponds to opening of presumed enhancer regions far upstream (Fig 2F). A similar pattern was observed for Sox2, with broadly accessibly chromatin near the TSS, but transcription being linked with ATAC peaks 453 kilobases upstream (SI Appendix, Fig S5). In the case of Sox2 these upstream peaks overlap with the start site of Sox2ot, a long non-coding RNA known to positively regulate Sox2 transcription (35) and thus may not represent a bona fide enhancer element. These data suggest glial cells preserve phenotypic plasticity in the postnatal period by maintaining accessible chromatin at transcription factor gene loci, thus 
permitting rapid activation of a neuroblast gene program by activating enhancers and other regulatory elements in the genome.

\section{Plp1-positive glial cells and neuroblasts in postnatal intestine maintain a transcriptional program} similar to embryonic neuronal progenitor cells

We and others have shown that postnatal glial cells can become neurons both in vivo and in vitro $(5,6$, $8,9)$. Additionally, glial cells are proliferative and express many marker genes of prenatal enteric neuronal precursors, such as Sox 10. We thus asked whether glial cells maintain a gene expression program closely matching embryonic enteric neuronal precursors. To test this, we took advantage of recently-published scRNA seq data of differentiating enteric neurons from the mouse small intestine at E15.5 and E18.5 (33). We integrated cells from both time points for our analysis. Recapitulating the authors' published results, we saw Sox10-expressing progenitor cells and Stmn2-expressing neurons along two predominant lineages, with a group of Sox 11 -expressing cells at an intermediate state between progenitors and neurons (Fig 3A).

Because our scRNA seq dataset was derived from Plpl-expressing cells and therefore does not include neurons, we could not transfer cell identities directly onto the embryonic dataset. We thus used a curated scRNAseq dataset of the mouse nervous system published by Zeisel et al (15). We first used canonical correlation to transfer cell identities from our scRNAseq data to glial cells in the Zeisel dataset, and then merged the Zeisel glial data with the same authors' neuronal dataset. To validate this approach, we identified marker genes for each cluster mapped from our data to the Zeisel data and saw an expression pattern very similar to our own data (SI Appendix, Fig S6A and S6B). This both confirms the validity of transferring cell labels using this approach and demonstrates the reproducibility of our enteric glial analysis. We then used canonical correlation to transfer cluster identities from the Zeisel dataset to the combined embryonic dataset. A large number of cells scored highly for S Phase and G2/M Phase clusters (Fig 3B, SI Appendix, Fig S7), which is consistent with highly proliferative nature of the prenatal ENS. We also noted that all cells in both lineages of developing neurons scored highly for neuronal identity (Fig 3B), which further validated our label transfer. The remainder of cells scored very highly for either neuroblasts or plastic glia from clusters 4 and 0 , respectively, in our scRNAseq data (Fig 3B). We examined expression of glial markers, neural stem cell-associated genes, and neuronal transcription factors in the assigned clusters in the embryonic dataset (Fig 3C). Similar to our scRNA seq dataset, we saw expression of glial markers in all cells except neurons, with higher transcription of Plpl in plastic glia (cluster 0) than in glial neuroblasts (cluster 4). Neuroblasts also expressed much higher levels of neural stem cell-associated genes, such as Sox 2 and Foxol, when compared to plastic glia (Fig 3C). Interestingly, S- and G2/M-phase cells more closely resembled neuroblasts than plastic glia in the embryo, while proliferative cells in the adolescent intestine are plastic glia (Fig 1C, Fig 3C). We conclude Plpl-expressing glial cells in the postnatal intestine maintain a transcriptional program highly similar to enteric neuronal progenitors.

\section{Motif enrichment and regulon activity implicate AP-1 transcription factors and inflammatory signaling in glial state transitions}

To understand the factors driving transitions between glial cell states, we used ChromVAR (29) to analyze motif enrichment patterns. Due to its small size, inter-sample variability, and unclear biological function, we excluded ATAC cluster 9 from this analysis. Motif enrichment patterns separated glial clusters and were reproducible across samples (Fig 4A). Similar to clustering based on ATAC peaks, we noted that glial neuroblasts (cluster 4) had a distinct set of motif enrichments, with transitional glial cells (cluster 5) showing a lesser degree of enrichment for the same motifs. Interestingly, cluster 6 of plastic glial cells appears to represent an intermediate state between transitional glial cells and other plastic glial clusters. As expected, neuroblasts are enriched for Phox2b, Hox gene, and Foxo1 motifs 
(Fig 4B). We observed that transitional cells (cluster 5) and some plastic glial cells (culture 6) are enriched for AP-1 transcription factor motifs, including Fos and Jun, and for the Nfkb1 motif (Fig 4C). To understand transcriptional control on a more global level, we used pySCENIC (28) to identify transcription factor regulons active in glial clusters. Consistent with the motif enrichment results, neuroblasts were heavily enriched for the Phox $2 \mathrm{~b}(+)$ regulon, as well as the Foxo1 $(+)$, Tbx $3(+)$, and Stat1(+) regulons (Fig 4D). Transitional glial cells were enriched for regulons controlled by the AP-1 factors Jun, Junb, Jund, and Fos, while regulons controlled by the NF-кB response elements Rel and Relb were active in cluster 6 (Fig 4D). We also noted that transcripts for the AP-1 factor genes Fos and Jun are enriched in snATACseq clusters 4, 5 and 6 (Fig 4E). While these results cannot determine the directionality of cell transitions, they implicate signal integration via AP-1 transcription factors and

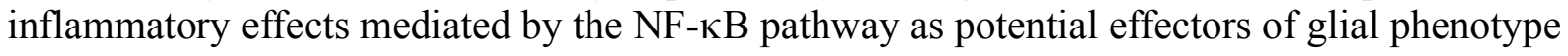
transitions.

\section{Suspension cultures of intestinal cells markedly enrich for neuroblasts and provide a niche for neurogenesis}

Our group and others have shown expansion of enteric glial cells in 3D culture with subsequent staining for neuronal markers after transfer to adherent culture in differentiation media $(5,9)$. However, these results could be confounded by neurons present in the initial cell population prior to culturing. To confirm whether glial cells expanded ex vivo retain neurogenic potential, we crossed Plp1::eGFP mice with Actl6b::Cre;(R)26-tdTomato mice to generate dual-reporter animals expressing eGFP in Plp1positive glial cells and permanently marking neurons with tdTomato expression. To confirm neurogenic potential of glial cells within these cultures, we sorted GFP+/tdTomato- cells and placed them in adherent culture. 24 hours after plating, all cells were GFP+ and no tdTomato + cells could be found (SI Appendix, Fig S8). However, after 5 days significant numbers of tdTomato + cells were observed (SI Appendix, Fig S8), thus confirming the emergence of neurons from a glial precursor population.

We then undertook scRNAseq and snATACseq of the 3D cultures. After 10 days in culture, we sorted the GFP+ (Plpl-expressing) cells from the total population. scRNAseq and multiomic sequencing was performed on the GFP+ fraction. We again used canonical correlation to score cells based on the populations of glial cells we identified in P14 glia (Fig 5A, 5B). Surprisingly, the overwhelming majority of GFP+ cells were assigned to either glial neuroblast or transitional glial groups, with only a small number of cells assigned to plastic glial cell clusters. A significant number of cells were also predicted to be neurons, and groups of cells were identified in the S and G2/M Phases of the cell cycle (Fig 5A). As expected, Plpl transcripts were detected in nearly all cells, although at diminished levels in glial neuroblasts and neurons (Fig 5C, 5D). Consistent with the assignment of most cells to glial neuroblast or transitional glial clusters, the glial neuroblast-associated transcripts Gfap, Sox2, Foxo1, $C d h 2$, and $T g f b 2$ are enriched in our 3D cultures (Fig 5C). These genes are enriched in S Phase and G2/ $\mathrm{M}$ Phase cells as well as in glial neuroblasts and transitional glial cells, suggesting the glial neuroblasts are not post-mitotic cells fully committed to neuronal fate (Fig 5C). This recapitulates the embryonic gut, where proliferating glia more closely resemble glial neuroblasts than plastic glial cells (Fig 3C).

The 3D cultures display the expected change in transcription factors along the neuronal lineage, with Sox 10 and Sox 2 declining markedly while Ascll and Phox $2 b$ increase (Fig 5D). Notably, Ascll remains high along the full trajectory of neuronal differentiation. This contrasts with embryonic enteric neurogenesis, during which Ascll expression peaks early during neuronal commitment before declining in fully differentiated neurons (33). This suggests the 3D culture niche may not permit terminal neuronal differentiation. Previous work has identified two main lineages of enteric neurons defined by the transcription factors Etvl and Bnc2 (33). We did observe an apparent bifurcation into these two groups (SI Appendix, Fig S9), albeit without emergence of the diverse subtypes of neurons previously 
identified along each lineage. As has been reported, we find that the Etv1 lineage contains Nos 1- and Vip-transcribing neurons, while the Bnc2 lineage is relatively enriched for Phox $2 a$ and Chat transcripts (SI Appendix, Fig S9). Regulon analysis also shows considerable overlap of transcriptional programs between 3D cultures and the embryonic ENS (SI Appendix, Fig S10A and S10B), although some differences are noted, such as activity of the Klf6(+) regulon in 3D cultures but not in embryonic ENS. Finally, we use RNA velocity analysis to identify the top 500 genes undergoing dynamic changes in expression within $3 \mathrm{D}$ cultures. Of these 500, $334(66.8 \%)$ were among the 5000 most variable genes in the combined embryonic dataset (Fig S11A, S1B). In both datasets, these 334 include genes marking neuronal progenitors (such as Sox10), those transiently expressed during neurogenesis (such as Sox 11), and neuronal marker genes (such as Snap25) (SI Appendix, Fig S11). Based on these data, we conclude that 3D cultures of LMMP-derived cells broadly recapitulate embryonic enteric neurogenesis without producing terminally differentiated enteric neurons.

We next used our 3D culture data to query whether AP-1 factors control the glial neuroblast phenotype. While our motif enrichment and regulon analyses implicate these factors in the transition between plastic glia and glial neuroblast states, directionality of the effect is not clear. If AP-1 factors are, as we hypothesize, critical for generating glial neuroblasts, we would anticipate high transcription of AP-1 genes within our 3D cultures. Conversely, if the AP-1 gene products function to push cells away from glial neuroblast status, 3D cultures that are heavily enriched for glial neuroblasts should not express AP-1 transcripts. Consistent with our hypothesis, transcripts for AP-1 factor genes Jun, Junb, Jund, Fos, and Fosb are enriched in transitional glial cells and glial neuroblasts within our 3D cultures (SI Appendix, Fig S12). Similarly, regulon analysis reveals strong enrichment for Jun $(+)$ and Fos $(+)$ regulons in 3D cultures (SI Appendix, Fig S9A and S9B). This activity correlates with Sox10 $(+)$, Sox2(+), and Foxd3(+) regulon activity. Moreover, AP-1 factor regulons are down-regulated along the trajectory of neurogenesis (SI Appendix, Fig S10B). Intriguingly, the same pattern is observed in the embryonic ENS (SI Appendix, Fig S10B). Taken together, these data implicate AP-1 transcription factors in promoting and maintaining the enteric neuroblast phenotype.

\section{Neuronal differentiation involves closing chromatin at glial-associated loci}

We next turned our attention to snATACseq data of 3D cultures. Utilizing 10X Genomics Multiome kits, we were able to obtain both gene expression and chromatin accessibility data from individual nuclei. We performed dimensional reduction and UMAP projection using snATACseq data, and subsequently used gene expression data to assign cell identities. We validated these cell identities by confirming expression of expected marker genes within each identity (SI Appendix, Fig S13). Consistent with our scRNAseq data, we found significant enrichment for transitional glial cells and glial neuroblasts, as well as dividing cells and differentiating neurons (Fig 5E). We took advantage of this to examine the chromatin changes occurring during neurogenesis. Neurons have closed chromatin at loci open in glial cells, while most of the peaks associated with neurons are also open in glial neuroblasts, albeit to a lesser degree (Fig 5F). This is illustrated by the Hoxa 5 locus, where the TSS and promoter show similar accessibility in neurons and in glial clusters (Fig 5G). In contrast, the Sox 10 and Plp1 loci show markedly diminished signal at gene bodies and promoters in neurons (Fig 5G and SI Appendix, Fig S14).

Transcription in the developing human ENS recapitulates the glial-to-neuronal trajectory Our work suggests cells with a glial gene expression program represent neuronal progenitors both during prenatal gut development and postnatally. We sought to determine whether this result is recapitulated in human biology. We examined a recently-published scRNAseq dataset of cells in the fetal human intestine (34). This dataset includes a group of cells distinct from the main population the authors call "Lymphoid Associated Glial" due to expression of GFRA3. We believe these cells actually 
represent Schwann cell precursors (SCPs), as they also express the canonical SCP marker genes $D H H$, $M A L$, and $M P Z$ (SI Appendix, Fig S15). Since SCPs are a separate neural crest lineage from the vagal neural crest, we excluded these cells from further analysis. Among the remaining 3962 cells, the authors annotated 12 clusters with identities inferred from gene expression (SI Appendix, Fig S16A). We noted the structure of the UMAP projection closely resembles the combined embryonic dataset, with a glial population connected to two distinct neuronal lineages by an intermediate bridging population. Glial cells express the expected marker genes, while the "ENS Progenitors" that comprise the bridge population have an intermediate transcriptional signature and are marked by high expression of ASCL1 (SI Appendix, Fig S16B). Similarly, we found glial cells and neurons to have distinct regulon activities, with the ASCL1-expressing "ENS Progenitors" displaying an intermediate complement of regulons (SI Appendix, Fig S16C). We further noticed that glial-associated regulons, such as SOX10(+), positively correlate with activity of AP-1 factor regulons, while neuronal regulons, such as PHOX2A $(+)$, were anticorrelated with AP-1 factor regulons (SI Appendix, Fig S16D, S17). This is consistent with our own results and other recent studies of murine ENS development $(33,36)$ and suggests the networks underlying ENS development are highly conserved among mammals.

\section{$\underline{\text { Discussion }}$}

This study integrates snATACseq and scRNAseq data to create a joint atlas of postnatal enteric glial cells. We identify numerous transcriptionally and epigenetically distinct subpopulations of myenteric glial cells, thus uncovering greater diversity than was previously appreciated. Our work shows most postnatal enteric glial cells maintain a chromatin structure permissive for phenotypic plasticity. This chromatin structure likely permits glial cells to rapidly undergo neurogenesis when appropriately stimulated. Glial subgroups include glial neuroblasts that are poised to become neurons, plastic glial cells that do not express neuroblast markers but maintain open chromatin at neuroblast and neuronal loci, and cells transitioning between states. We show that glial neuroblasts can be expanded in ex vivo $3 \mathrm{D}$ cultures while retaining differentiation potential and may thus represent a valuable source of autologous cells to treat neurointestinal disorders.

Prior studies have utilized immunostaining and functional assays to demonstrate enteric glial heterogeneity (10-12). Recent efforts at profiling the entire mouse nervous system and the human enteric nervous system have included enteric glial cells $(14,15)$ and have reported multiple clusters of glial cells based on differential expression of highly variable genes. However, these prior scRNAseq studies do not include detailed analyses of glial subtypes. Our work expands upon these reports in several ways. First, multiomic data allows us to assess both gene expression and epigenetic regulation. This approach has uncovered a molecular basis for glial phenotypic plasticity, as glial cells maintain open chromatin at gene loci that control neurogenesis. Chromatin closes at glial marker loci when neuroblasts become neurons, presumably to prevent neurons from reverting to a glial fate. Secondly, our work suggests there are two main types of glial cells in the adolescent mouse ENS - neuroblasts marked by Gfap transcription and plastic glial cells not transcribing Gfap but maintaining open chromatin at neuroblast and neuronal gene loci. The latter group expresses genes associated with ECM remodeling and ion transport, as previously reported in bulk RNA sequencing studies (10). We speculate these cells, which we have named "plastic glia," have ECM and fluid ion balance roles in addition to functioning as a reservoir of potential neurons. In addition to these two groups, we also identify proliferative glial cells, cells apparently in transition between plastic and neuroblast programs, and a small group of "restricted" glial cells with inaccessible chromatin at genomic loci associated with the neuroblast phenotype. Correlation between our scRNAseq data and the scRNAseq data published by Zeisel et al shows significant overlap, validating the glial cell diversity reported by our two groups. 
Combined analysis of transcription factor binding motifs and gene expression in our data implicates AP-1 transcription factors such as Jun and Fos and the NF- $\kappa$ B pathway in facilitating transitions between the neuroblast phenotype and other glial cell types. This result is consistent with prior reports of inflammation inducing neurogenesis from myenteric glial cells $(5,8,9)$. AP-1 factors and NF- $\mathrm{B}$ family genes are immediate early response genes induced quickly when cells are stimulated $(37,38)$. AP-1 factors coordinate cell type-specific responses by cooperating with cell identity transcription factors to select enhancers and alter gene expression (39). NF- $\mathrm{KB}$ signaling, meanwhile, can crossactivate AP-1 gene expression $(40,41)$. We thus speculate that some glial cells lie in a non-neuroblastic state when unstimulated. Inflammation or other signals trigger an AP-1 response, and AP-1 factors in turn cooperate with glial-specific transcription factors to remodel chromatin and activate neuroblast enhancers. This model is consistent with stimulation of Gfap and Fos transcription when myenteric glial cells are challenged with lipopolysaccharide $(13,42-44)$. The high expression of AP-1 genes (Fos, Jun, etc) in glial neuroblasts, but not neurons, within our suspension cultures also supports this model, as does the correlation of AP-1 factor expression and neuronal progenitor factor expression we identified in the prenatal ENS of both humans and mice. Induction of immediate-early response genes, such as the AP-1 factors, is a recognized consequence of cell processing for scRNAseq (45) that may confound this result. However, the immediate-early gene response due to tissue processing occurs across cell types (46), while in our data AP-1 factor expression is limited to a subset of glial cells and declines markedly in differentiating neurons. Moreover, the reproducible correlation between neuroblast genes (Gfap, Sox2, Foxo1, etc) and AP-1 genes in datasets from several laboratories and different biological contexts (embryonic, postnatal adolescent, and 3D culture) argues in favor a genuine biologic effect.

Successful cell therapies for ENS disorders will require three conditions be met: 1) engraftment and survival of the therapeutic product in the recipient, 2) functional integration of therapeutic cells with the host ENS, and 3) long-term safety of the therapeutic cells. Neurons are postmitotic and challenging to isolate, making neuronal progenitors a more suitable therapeutic product. Our results suggest enteric glia as a promising cell source. However, given the highly proliferative nature of ex vivo glial cultures, long-term follow-up will be critical to ensure the cells do not proliferate indefinitely after implantation. While we demonstrate neuronal differentiation that recapitulates many of the transcriptional events occurring during prenatal ENS development, we did not observe emergence of all enteric neuronal subtypes. We also observe persistent expression of several factors that decline in mature neurons, such as Ascll (33). Ensuring that neurons derived from ex vivo glial cultures are capable of forming terminally differentiated neurons in the correct proportions after implantation will be critical prior to therapeutic application.

We demonstrate significant transcriptional similarity between postnatal glial cells and prenatal ENS progenitors. The transcriptional changes postnatal glial cells undergo as they become neurons closely matches changes identified during embryonic ENS development $(33,36)$, suggesting we should abandon the notion of distinct neuronal and glial lineages derived from a common precursor. We find that glial cells express many genes associated with embryonic ENS progenitors, including transcription factors such as Sox10 and Sox6, the ubiquitin ligase Fbxo7, and Plp1 (33). scRNAseq studies of the prenatal mouse ENS have all identified a differentiation trajectory from cells expressing glial genes toward neurons, but a distinct glial lineage has not been convincingly demonstrated $(33,36)$. We propose a new model of common neural crest-derived progenitors giving rise to plastic glial cells and glial neuroblasts, which in turn give rise to terminally-differentiated neurons (Fig 6). We believe these glial subtypes persist postnatally and represent the EGCs that have been observed for many years. Our data also identify a postnatal glial subgroup marked by closed chromatin at neuronal-associated loci that we have called "restricted glia" and that likely lacks neuronal potential (Fig 6). 
Our study is limited in several respects. We only assessed glial cells in the muscularis propria, so our findings cannot be extended to submucosal glia or to the Schwann cells accompanying extrinsic nerves. Characterizing submucosal glia and Schwann cells, and correlating gene expression to biological functions, will be an important area for future work. In addition, we only assessed adolescent mice approximately two weeks after birth, at a stage when murine ENS development is ongoing $(47,48)$. Changes to glial cells during early adulthood and later in life are beyond the scope of this report but may be important. Despite the transcriptional similarity between prenatal ENS precursors and postnatal myenteric glial cells, we were unable to compare epigenetic signatures of these populations. Exploring chromatin dynamics during embryonic ENS development will be important to understanding specification of enteric neurons. Finally, although this work greatly extends our understanding of myenteric plexus glial diversity, rigorous experimental models will be necessary to test the many hypotheses we have generated. Despite these limitations, this atlas of glial cells from the small intestine of adolescent mice represents a novel, publicly-available resource we anticipate will be of great value to the research community and may facilitate efforts to develop autologous, cell-based therapies for neurointestinal diseases.

\section{Acknowledgments}

We thank David Pepin, Marie Meinsohn, and Phong Nguyen for their assistance preparing single-cell gene expression libraries. Special thanks to Patricia K. Donahoe for valuable feedback on the manuscript. This work is supported by National Institutes of Health grants F32DK121440 to RAG and R01DK103785 and R01DK119210 to AMG.

\section{References}

1. N. Nagy, A. M. Goldstein, Enteric nervous system development: A crest cell's journey from neural tube to colon. Seminars in Cell \& Developmental Biology 66, 94-106 (2017).

2. J. B. Furness, The enteric nervous system and neurogastroenterology. Nature Reviews Gastroenterology \& Hepatology 9, 286-294 (2012).

3. J. B. Furness, The enteric nervous system: normal functions and enteric neuropathies. Neurogastroenterol. Motil. 20 Suppl 1, 32-38 (2008).

4. A. J. Burns, et al., White paper on guidelines concerning enteric nervous system stem cell therapy for enteric neuropathies. Developmental Biology 417, 229-251 (2016).

5. N. M. Joseph, et al., Enteric glia are multipotent in culture but primarily form glia in the adult rodent gut. 14 .

6. C. Laranjeira, et al., Glial cells in the mouse enteric nervous system can undergo neurogenesis in response to injury. J Clin Invest 121, 3412-3424 (2011).

7. R. Hotta, et al., Delivery of enteric neural progenitors with 5-HT4 agonist-loaded nanoparticles and thermosensitive hydrogel enhances cell proliferation and differentiation following transplantation in vivo. Biomaterials 88, 1-11 (2016).

8. J. Belkind-Gerson, et al., Colitis promotes neuronal differentiation of Sox2+ and PLP1+ enteric cells. Scientific Reports 7, 2525 (2017). 
9. J. Belkind-Gerson, et al., Colitis Induces Enteric Neurogenesis Through a 5-HT4-dependent Mechanism: Inflammatory Bowel Diseases 21, 870-878 (2015).

10. M. Rao, et al., Enteric glia express proteolipid protein 1 and are a transcriptionally unique population of glia in the mammalian nervous system. Glia 63, 2040-2057 (2015).

11. W. Boesmans, R. Lasrado, P. Vanden Berghe, V. Pachnis, Heterogeneity and phenotypic plasticity of glial cells in the mammalian enteric nervous system. Glia 63, 229-241 (2015).

12. N. Maudlej, M. Hanani, Modulation of dye coupling among glial cells in the myenteric and submucosal plexuses of the guinea pig. Brain Research 578, 94-98 (1992).

13. C. Rosenbaum, et al., Activation of Myenteric Glia during Acute Inflammation In Vitro and In Vivo. PLOS ONE 11, e0151335 (2016).

14. E. Drokhlyansky, et al., The Human and Mouse Enteric Nervous System at Single-Cell Resolution. Cell, S0092867420309946 (2020).

15. A. Zeisel, et al., Molecular Architecture of the Mouse Nervous System. Cell 174, 999-1014.e22 (2018).

16. M. Rao, M. D. Gershon, Enteric nervous system development: what could possibly go wrong? Nat Rev Neurosci 19, 552-565 (2018).

17. J. I. Lake, R. O. Heuckeroth, Enteric nervous system development: migration, differentiation, and disease. American Journal of Physiology-Gastrointestinal and Liver Physiology 305, G1-G24 (2013).

18. J. Kim, L. Lo, E. Dormand, D. J. Anderson, SOX10 Maintains Multipotency and Inhibits Neuronal Differentiation of Neural Crest Stem Cells. Neuron 38, 17-31 (2003).

19. S. Elworthy, J. P. Pinto, A. Pettifer, M. L. Cancela, R. N. Kelsh, Phox2b function in the enteric nervous system is conserved in zebrafish and is sox10-dependent. Mechanisms of Development 122, 659-669 (2005).

20. T. Y. Y. Leon, et al., Transcriptional regulation of RET by Nkx2-1, Phox2b, Sox10, and Pax3. Journal of Pediatric Surgery 44, 1904-1912 (2009).

21. D. Lang, J. A. Epstein, Sox10 and Pax3 physically interact to mediate activation of a conserved cRET enhancer. Human Molecular Genetics 12, 937-945 (2003).

22. G. M. Kruger, et al., Neural Crest Stem Cells Persist in the Adult Gut but Undergo Changes in Self-Renewal, Neuronal Subtype Potential, and Factor Responsiveness. Neuron 35, 657-669 (2002).

23. B. S. Mallon, W. B. Macklin, Overexpression of the 3'-Untranslated Region of Myelin Proteolipid Protein mRNA Leads to Reduced Expression of Endogenous Proteolipid mRNA. Neurochem Res 27, 1349-1360 (2002).

24. M. Zou, H. Luo, M. Xiang, Selective neuronal lineages derived from Dll4-expressing progenitors/ precursors in the retina and spinal cord. Dev Dyn 244, 86-97 (2015). 
25. L. Madisen, et al., A robust and high-throughput Cre reporting and characterization system for the whole mouse brain. Nat Neurosci 13, 133-140 (2010).

26. C. Hafemeister, R. Satija, Normalization and variance stabilization of single-cell RNA-seq data using regularized negative binomial regression. Genome Biology 20, 296 (2019).

27. V. D. Blondel, J.-L. Guillaume, R. Lambiotte, E. Lefebvre, Fast unfolding of communities in large networks. J. Stat. Mech. 2008, P10008 (2008).

28. B. Van de Sande, et al., A scalable SCENIC workflow for single-cell gene regulatory network analysis. Nat Protoc 15, 2247-2276 (2020).

29. A. N. Schep, B. Wu, J. D. Buenrostro, W. J. Greenleaf, chromVAR: inferring transcription-factorassociated accessibility from single-cell epigenomic data. Nat Methods 14, 975-978 (2017).

30. O. Fornes, et al., JASPAR 2020: update of the open-access database of transcription factor binding profiles. Nucleic Acids Research 48, D87-D92 (2020).

31. G. L. Manno, et al., RNA velocity of single cells. Nature 560, 494-498 (2018).

32. V. Bergen, M. Lange, S. Peidli, F. A. Wolf, F. J. Theis, Generalizing RNA velocity to transient cell states through dynamical modeling. bioRxiv, 820936 (2019).

33. K. Morarach, et al., Diversification of molecularly defined myenteric neuron classes revealed by single-cell RNA sequencing. Nature Neuroscience 24, 34-46 (2021).

34. D. Fawkner-Corbett, et al., Spatiotemporal analysis of human intestinal development at single-cell resolution. Cell 184, 810-826.e23 (2021).

35. Y. Zhan, et al., Long non-coding RNA SOX2OT promotes the stemness phenotype of bladder cancer cells by modulating SOX2. Molecular Cancer 19, 25 (2020).

36. S.-T. Lau, et al., Activation of Hedgehog Signaling Promotes Development of Mouse and Human Enteric Neural Crest Cells, Based on Single-Cell Transcriptome Analyses. Gastroenterology 157, 1556-1571.e5 (2019).

37. W. W. Lamph, P. Wamsley, P. Sassone-Corsi, I. M. Verma, Induction of proto-oncogene JUN/AP1 by serum and TPA. Nature 334, 629-631 (1988).

38. W. Du, I. Mills, B. E. Sumpio, Cyclic strain causes heterogeneous induction of transcription factors, AP-1, CRE binding protein and NF-kB, in endothelial cells: species and vascular bed diversity. J Biomech 28, 1485-1491 (1995).

39. T. Vierbuchen, et al., AP-1 Transcription Factors and the BAF Complex Mediate SignalDependent Enhancer Selection. Molecular Cell 68, 1067-1082.e12 (2017).

40. S. Fujioka, et al., NF-кB and AP-1 Connection: Mechanism of NF-кB-Dependent Regulation of AP-1 Activity. Mol Cell Biol 24, 7806-7819 (2004). 
41. Z. Ji, L. He, A. Regev, K. Struhl, Inflammatory regulatory network mediated by the joint action of NF-kB, STAT3, and AP-1 factors is involved in many human cancers. PNAS 116, 9453-9462 (2019).

42. C. Cirillo, et al., Proinflammatory stimuli activates human derived enteroglial cells and induces autocrine nitric oxide production. Neurogastroenterology \& Motility 23, e372-e382 (2011).

43. Y. Nasser, C. M. Keenan, A. C. Ma, D.-M. McCafferty, K. A. Sharkey, Expression of a functional metabotropic glutamate receptor 5 on enteric glia is altered in states of inflammation. Glia 55, 859-872 (2007).

44. G. B. T. von Boyen, et al., Proinflammatory cytokines increase glial fibrillary acidic protein expression in enteric glia. Gut 53, 222-228 (2004).

45. S. C. Van Den Brink, et al., Single-cell sequencing reveals dissociation-induced gene expression in tissue subpopulations. Nature Methods 14, 935-936 (2017).

46. Y. E. Wu, L. Pan, Y. Zuo, X. Li, W. Hong, Detecting Activated Cell Populations Using Single-Cell RNA-Seq. Neuron 96, 313-329.e6 (2017).

47. J. P. P. Foong, Postnatal Development of the Mouse Enteric Nervous System. Adv Exp Med Biol 891, 135-143 (2016).

48. P. Parathan, Y. Wang, A. JL. Leembruggen, J. C. Bornstein, J. PP. Foong, The enteric nervous system undergoes significant chemical and synaptic maturation during adolescence in mice. Developmental Biology 458, 75-87 (2020). 
A)

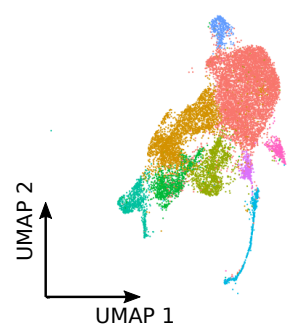

- 0 - Plastic glia

1 - Transitional glia

- 2 - Environmental response

- 3 - Transitional glia

- 4 - Glial neuroblast

- 5 - G2/M phase

6 - Restricted glia

7 - Plastic glia

8 - S phase

UMAP 1

B)

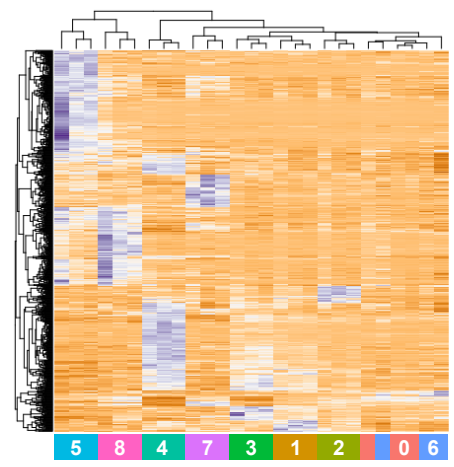

C)

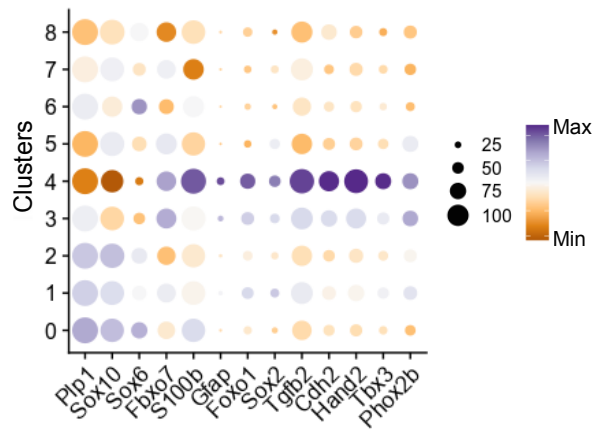

snATACseq Dataset

D)

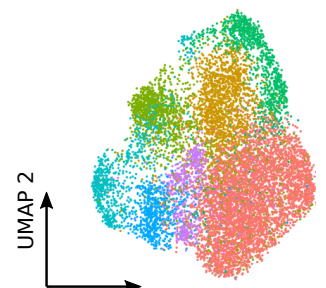

UMAP 1
0 - Plastic glia

1 - Plastic glia

- 2 - Plastic glia

- 3 - Restricted glia

- 4 - Glial neuroblast

- 5 - Transitional glia

6 - Plastic glia

9 - Unknown
E)

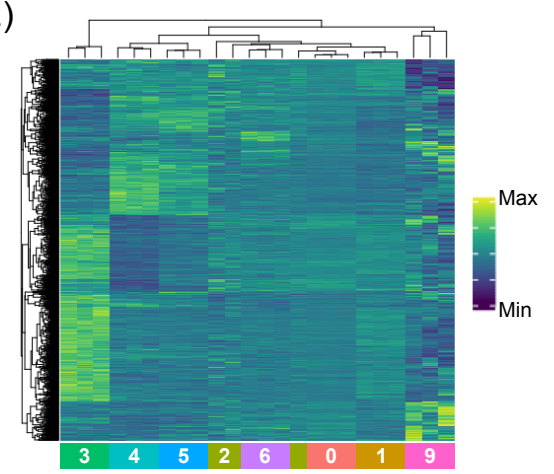

F)

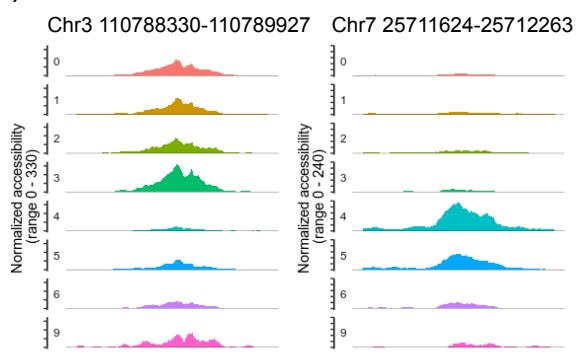

G)

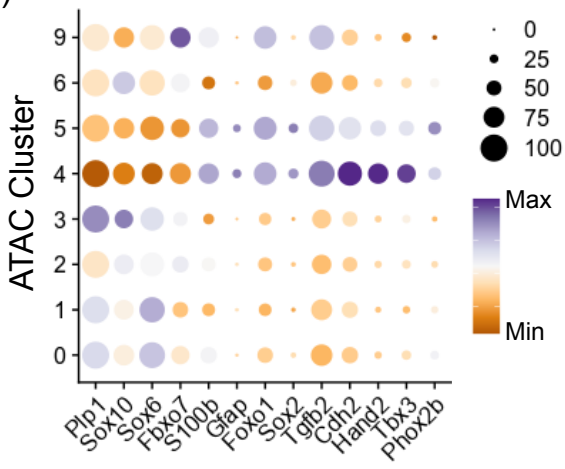

H)

Predicted Identity

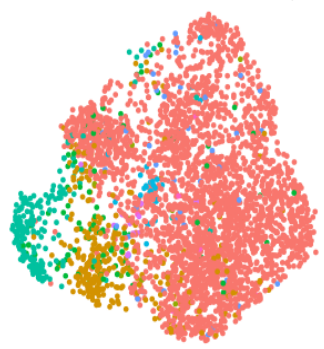

0 - Plastic glia

- 1 - Transitional glia

- 2 - Environmental response

- 3 - Transitional glia

- 4 - Glial neuroblast

5 - G2/M phase

- 6 - Restricted glia

7 - Plastic glia

8 - S phase
I)

Cluster 0

Cluster 4

score

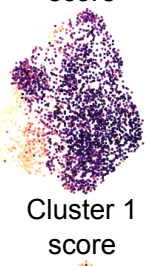

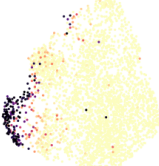

Cluster 3 score
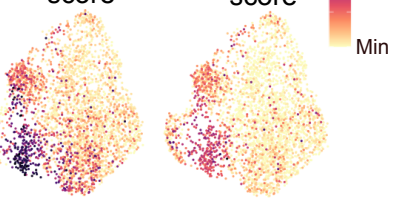
A) Glial Neuronal $\quad$ Max

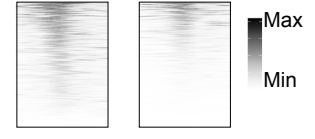

B)
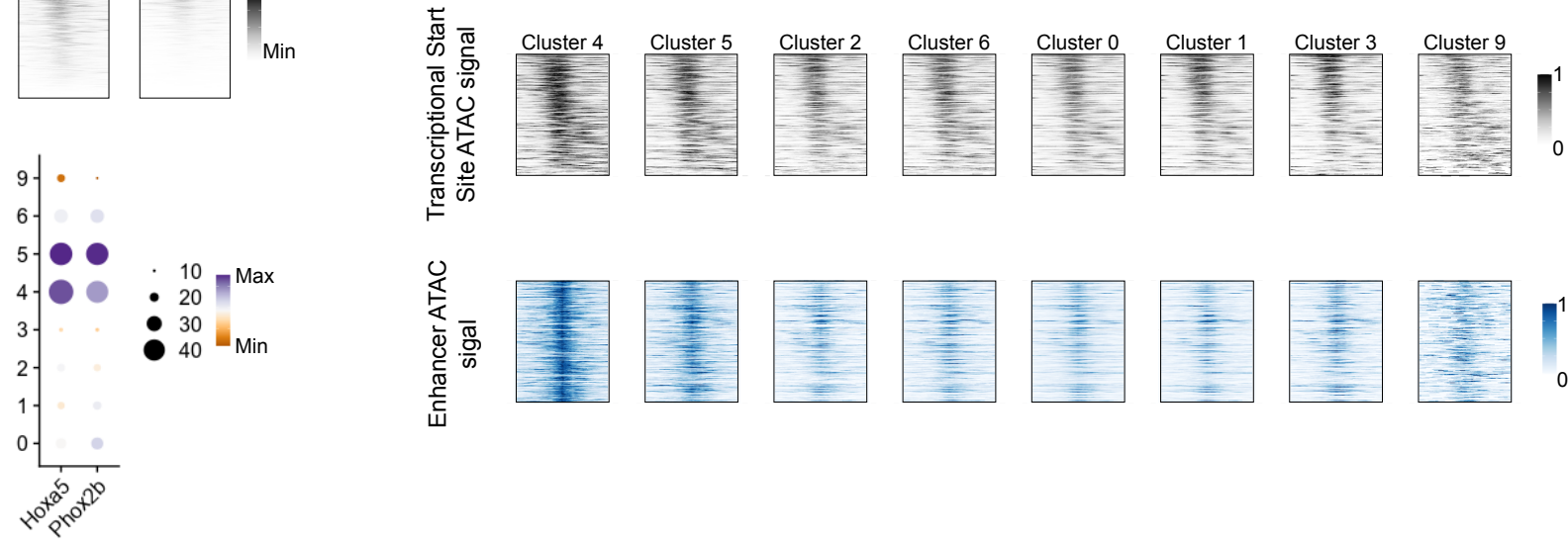

C)

Phox2b

D)
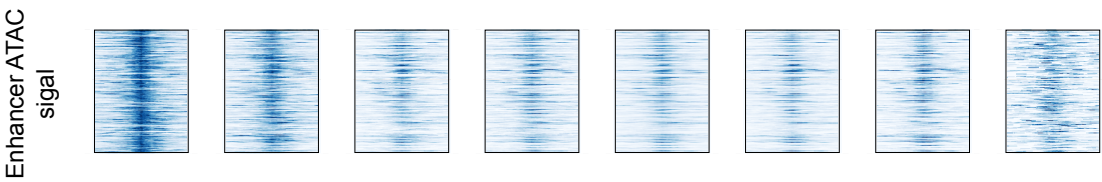

0
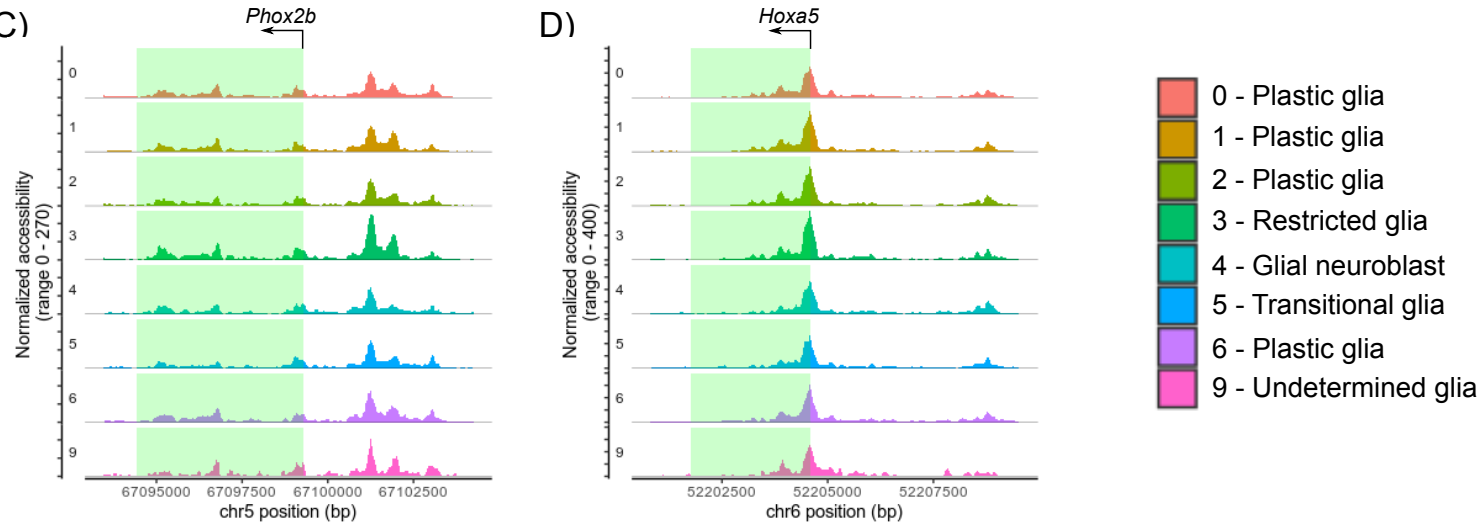

F)

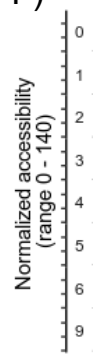

号
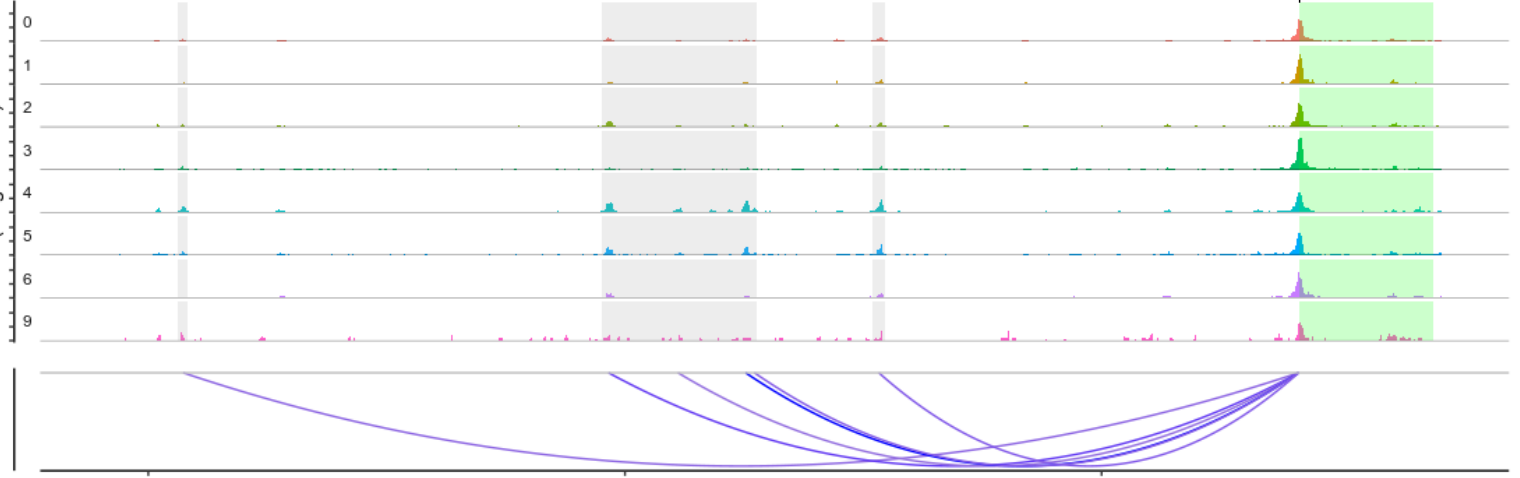
A)
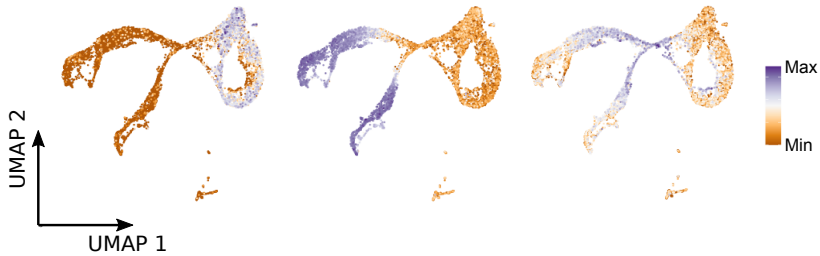

\section{Predicted Identity}

\section{B)}

C)
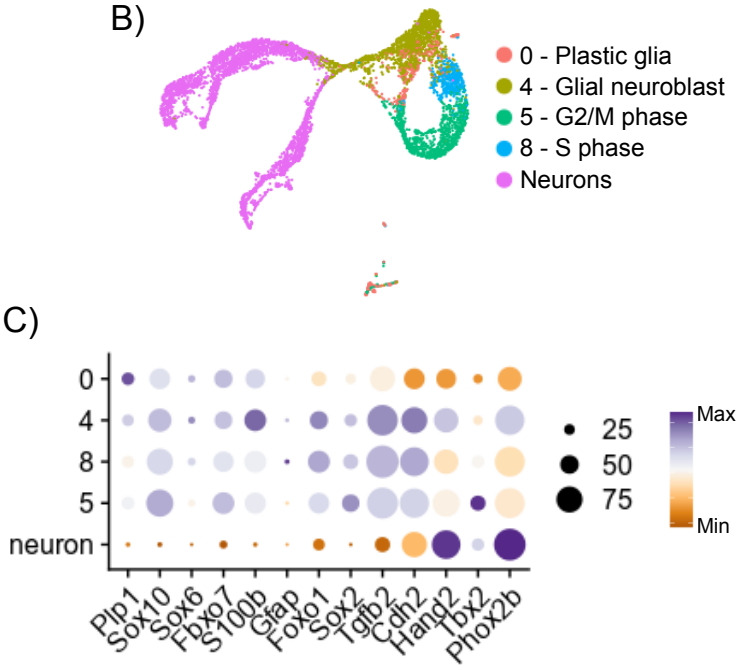
A

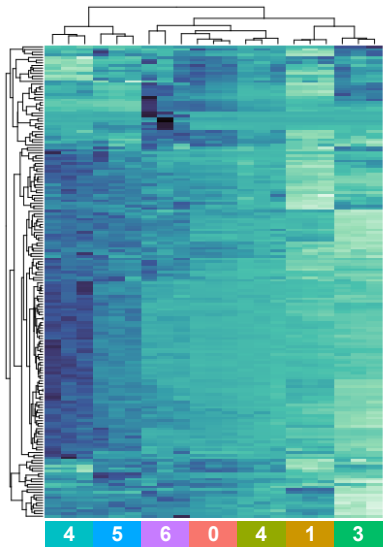

D)

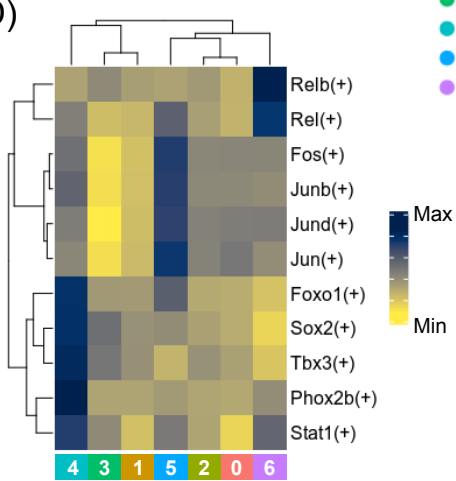

B) $\begin{array}{cc}{ }_{-1}^{6} & \\ -4 & \\ -2 & \\ -0 & \\ -2 & \\ -4 & \mathrm{n}\end{array}$

0 - Plastic glia - 1 - Plastic glia - 2 - Plastic glia - 3 - Restricted glia - 4 - Neuroblast 5 - Transitional - 6 - Plastic glia
6 - Plastic glia

5 - Transitional

4 - Neuroblast

3 - Restricted glia

2 - Plastic glia

1 - Plastic glia

0 - Plastic glia
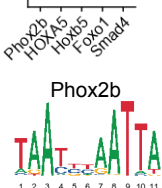

HOXA5

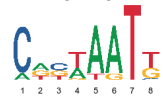

Hoxb5

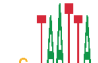

A I D I I C A
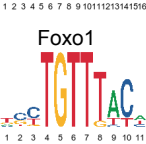

Smad4

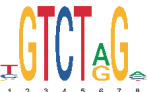

C)

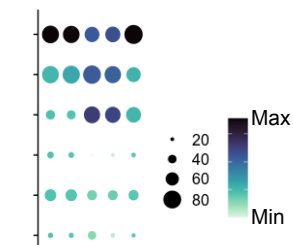

- $-\circ$

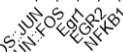

FOS::JUN

GACTCA

JUN::FOS

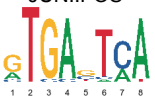

Egr1

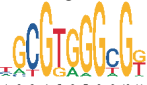

EGR2

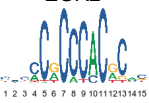

NFKB1

E)

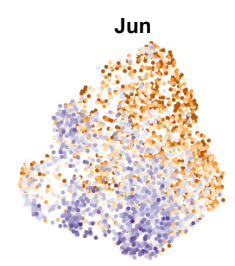

Fos

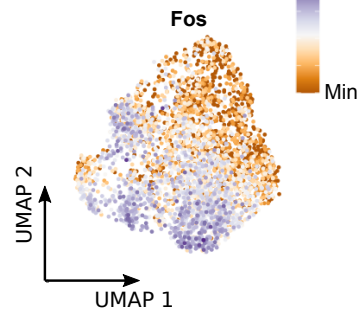


A)

\section{Predicted ID}

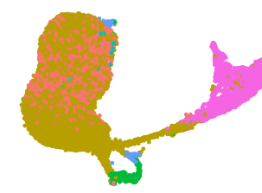

1 - Transitional glia

- 4 - Glial neuroblast

- 5- G2/M Phase

- 7 - Plastic glia

- 8 -S Phase

- Neuron

C)

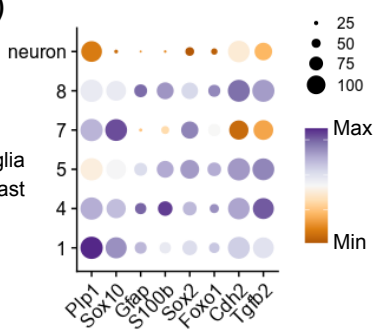

B)

Neuroblast Score

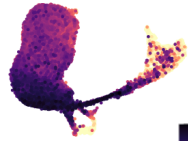

D)

Neuron Score

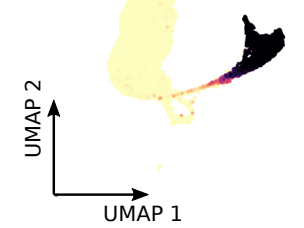

E)

Predicted ID

Predicted ID F)

Neuron

- 4 - Glial neuroblast

5 - G2/M Phase

- 1 - Transitional glia

UMAP 1

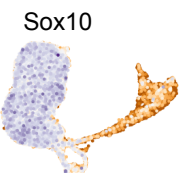

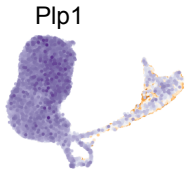

Phox2b

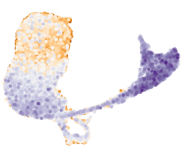

G)

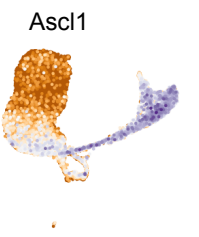

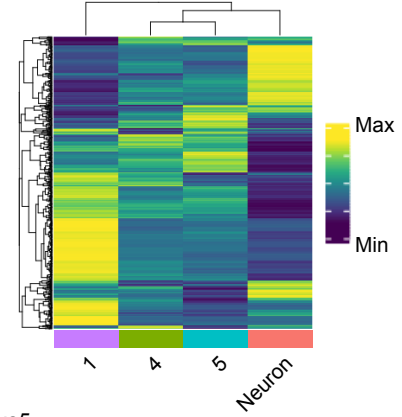

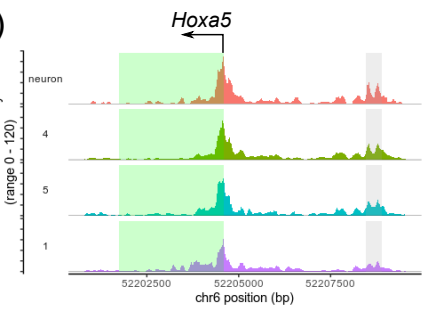

Sox10

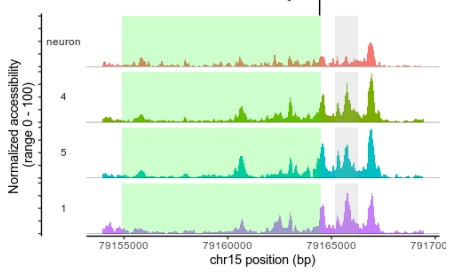




\section{Traditional Model}

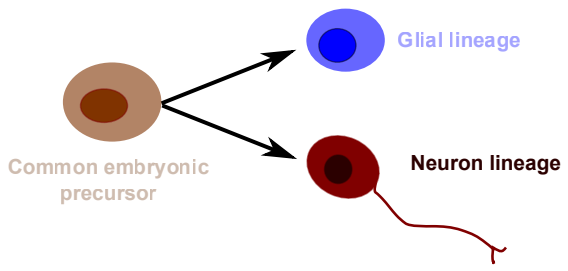

\section{Revised Model}
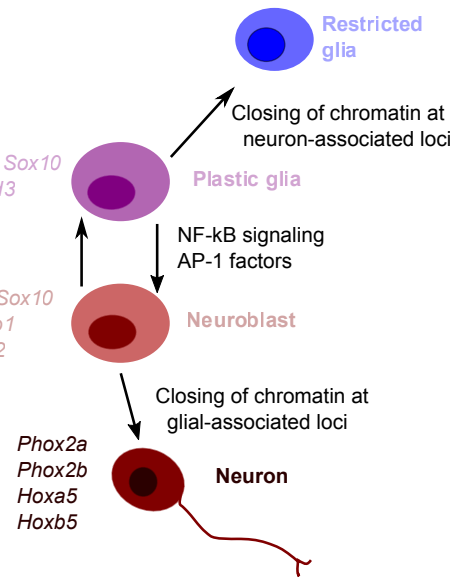